\title{
6 Benchmarking: Best practice als Vorbild
}

Christine Kuch

Benchmarking kann als eine der besonders motivierenden Methoden zum Anstoßen von Verbesserungsprozessen in Organisationen gelten. Denn beim Benchmarking vergleicht man Prozesse bzw. Ergebnisse der eigenen Organisation mit den Prozessen bzw. Ergebnissen anderer Organisationen anhand zuvor definierter Indikatoren. Zielsetzung dabei ist es, von denjenigen zu lernen, die im Vergleich die besten Ergebnisse, die Benchmarks, erzielen. Man erfährt im Benchmarking also nicht nur mögliche Verbesserungsoptionen, sondern auch die Bedingungen, die für das Erreichen der Verbesserungen von Bedeutung sind. Kurz gefasst ist Benchmarking Lernen von Best Practice.

Der Ablauf eines Benchmarking-Prozesses stellt sich prinzipiell wie folgt dar:

1. Entwicklung eines Kennzahlen-Sets, dem alle Beteiligten zustimmen

2. Erhebung der Daten in den teilnehmenden Organisationen

3. Auswertung der Daten

4. Rückmeldung der Ergebnisse der eigenen Organisation im Vergleich zu Ergebnissen anderer Organisationen. Meist werden diese im Vergleich zu den durchschnittlich erzielten Ergebnissen und zum besten Ergebnis gesetzt. Zumeist werden die anderen Organisationen anonymisiert dargestellt.

5. Austausch mit und Lernen von den Besten: Was machen sie anders? Was können wir davon auf unsere Organisation übertragen?

Meistens wird der fünfte Schritt nicht umgesetzt, teils weil die Teilnehmenden sich nur unter der Bedingung der Anonymität beteiligen, teils aufgrund von bestehendem Wettbewerb zwischen den Beteiligten. Allerdings wird vielfach berichtet, dass schon allein die Rückmeldung der Ergebnisse positive 
Effekte erzielten. Die Erkenntnis, in einem Bereich vergleichsweise weniger gut zu sein als man es angenommen hat, kann deutlich zu Veränderungen motivieren. Ein diesbezüglich interessanter Bericht ist einem Interview des für die Messung der Ergebnisqualität von schweizerischen Spitälern zuständigen Vereins Outcome zu entnehmen (s. Links).

\section{Aufgabe 9 für anwendungsfreudige Leser}

Überlegen Sie im Team bzw. im Vorstand:

- Zu welchen Themen wäre für uns ein Benchmarking interessant?

- Wem fallen welche Praxen/Ärzteverbünde ein, mit dem man einen Benchmarking-Prozess zum Thema xy initiieren könnte?

- Wer nimmt das Projekt federführend in die Hand?

Die einzelnen Ablaufschritte eines Benchmarking-Prozesses werden nachstehend ausgeführt.

Zur Entwicklung des Kennzahlen-Sets kann auf die beiden vorangehenden Kapitel zu den Themen Prozessmessung bzw. Outcomemessung (s. Kap. III. 4 und III.5) verwiesen werden, mit der Besonderheit, dass sich hier nicht nur Beteiligte einer Praxis auf „sinnvolle“ Messbereiche und -indikatoren einigen müssen, sondern Beteiligte mehrerer Gesundheitsorganisationen. Dass dies kein leichtes Unterfangen ist, dürfte unmittelbar klar sein: Schließlich muss die Messung so ökonomisch durchführbar wie nur irgend möglich sein, um den Aufwand möglichst gering zu halten. Zugleich braucht man für die relevanten Bereiche aussagekräftige und änderungssensitive Indikatoren, die allgemein anerkannt, nach Möglichkeit evidenzbasiert sind. Bei medizinischen Indikatoren empfiehlt es sich, auf vorhandene Entwicklungen zurückzugreifen, oder diese zumindest bei der Entwicklung neuer Indikatoren-Sets zu berücksichtigen. Neue Indikatoren-Sets müssen in einem Prä-Test erprobt werden, damit zumindest die gröbsten Anpassungen vorgenommen werden können, bevor die Messung in die Routine übergeht. Beispiele für mögliche Indikatoren sind in den Kapiteln III.4 und III.5, Prozessmessung und Outcomemessung, zu entnehmen. Gerade unter Benchmarking-Aspekten empfiehlt es sich, über rein medizinische Indikatoren hinaus auch Indikatoren für die Unternehmensführung zu integrieren (s. Kap. III.6).

Innerhalb von Netzen kann die Etablierung eines Benchmarkings zudem zur Unterstützung einer verbindlichen Umsetzung von Behandlungspfaden genutzt werden, beispielsweise indem die individuelle Erfüllung der jeweilig vereinbarten Vertragskriterien im Vergleich zu den anderen teilnehmenden Praxen zurückgemeldet wird. Zudem können sich Praxen auch in Bezug auf den erzielten Entwicklungsstand beispielsweise bei der Umsetzung von QEP, EFQM oder anderer QM-Systeme netzintern vergleichen, in Bezug auf den organisationalen Entwicklungsstand mit der Internationalen Maturity Matrix, auch mit Praxen aus anderen europäischen Ländern. 
Die Erhebung der Daten sollte, wie schon angemerkt, so ökonomisch wie möglich gestaltet sein. Dabei ist jedoch darauf zu achten, dass die Messung in den teilnehmenden Praxen in der gleichen Art und Weise erfolgt, da sonst nachher Äpfel mit Birnen verglichen werden und dies würde zu unbefriedigenden Ergebnissen führen. Zu diesem Zweck muss die Datenerhebung nach Möglichkeit standardisiert werden, zumindest was die Messzeitpunkte und die Messverantwortlichen angeht. Es ist auch sinnvoll, neben den Indikatoren im engeren Sinne weitere Daten, die die Messergebnisse beeinflussen können, wie z. B. Art und Umfang des Patientenklientels, Größe der Praxis, usw. mit zu erheben. Für die Organisation eines Benchmarkings in einem Verbund empfiehlt es sich, eine unterstützende zentrale Hotline einzurichten, bei der man sich Hilfe bei entstehenden Fragen holen kann. Für ein selbst organisiertes Benchmarking unabhängiger Praxen ist es lohnenswert, beispielsweise in einem Qualitätszirkel Probleme, die bei der Messung auftreten, regelmäßig zu besprechen und so die Qualität der Messung nach und nach zu optimieren.

Für die Auswertung der Daten ist vor allem auf die methodische Qualität und die zu leistende Vertraulichkeit der auswertenden Institution $\mathrm{zu}$ achten. Es kann daher auch bei selbst organisierten Benchmarking-Prozessen sinnvoll sein, zu diesem Zweck ein externes Institut zu beauftragen, damit die Anonymität glaubhaft gewährleistet bleibt. Zudem muss sichergestellt werden, dass die Auswertung der Daten transparent, nachvollziehbar und objektiv erfolgt.

Es hat sich bewährt, die Rückmeldung der Ergebnisse visuell zu unterstützen. Grafiken, die „mein Ergebnis“im Vergleich zum Besten und zum Durchschnitt zeigen, sind hilfreich. Je nach Indikator kann die Ausweisung der Konfidenzintervalle sinnvoll sein. Abbildung 6 zeigt einige Möglichkeiten, Benchmarking-Daten grafisch aufzubereiten.

Soll der Benchmarking-Prozess an dieser Stelle beendet werden, ist eine rein schriftliche Rückmeldung ausreichend, wobei Möglichkeiten zu Rückfragen an die auswertende Stelle eingerichtet werden sollten. Um einen nachhaltigen Lernprozess zu etablieren, sollten die Erhebungen in einem zumindest jährlichen Abstand wiederholt werden. Auf diese Weise können die einzelnen Praxen ihre Fortschritte erkennen und man kann insgesamt erfassen, ob der Benchmarking-Prozess tatsächlich zu einer Verbesserung bei den Teilnehmenden führt.

Ist auch ein konkreter Austausch mit den Besten möglich, können beispielsweise in einem praxisübergreifenden Qualitätszirkel die Besten ihre Ergebnisse offenlegen und berichten, welche Faktoren aus ihrer Sicht zu diesem guten Ergebnis beigetragen haben. Wichtig dabei ist, dass Dinge meist nicht einfach „kopiert“ werden können („Ab heute läuft das genau so wie in der Praxis von Dr. Schmitz“), sondern immer auf die eigene Organisation mit ihren individuellen Bedingungen übertragen werden müssen („Wie wollen wir das, was bei Dr. Schmitz gut funktioniert, auf unsere Praxis übertragen?“). 


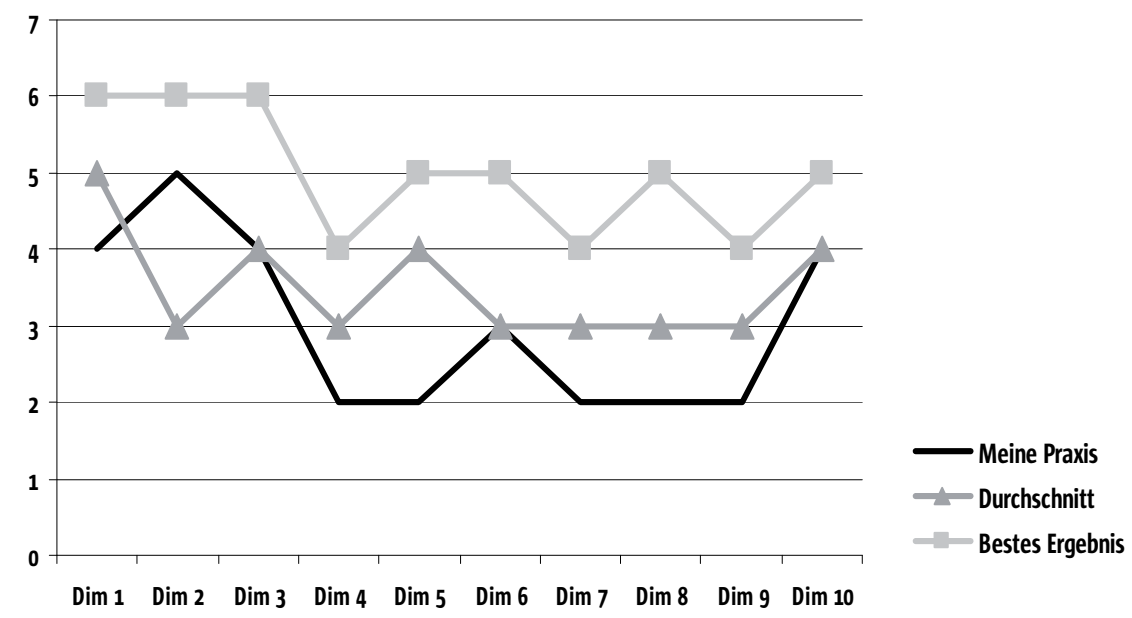

\section{Zufriedenheitsscore}

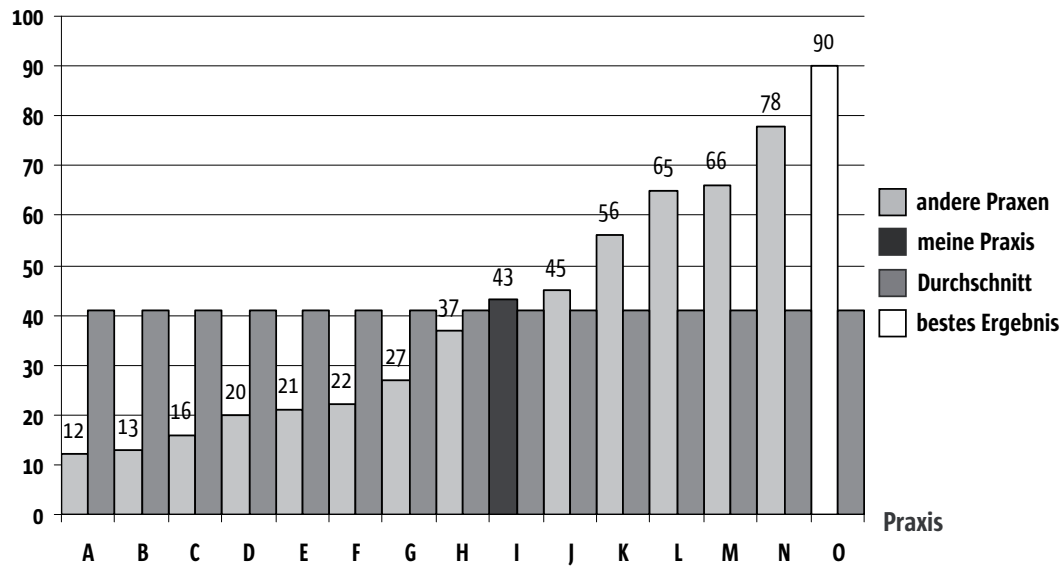

Abb. 6 Grafische Darstellung von Benchmarking-Daten

Im Verbund können zentrale, nach Möglichkeit moderierte Veranstaltungen mit den „Besten“ initiiert werden, bei denen Interessierte über erforderliche und sinnvolle Bedingungen für erzielte Ergebnisse diskutieren.

\section{Link}

Erfahrungsbericht zur den Messungen im Verein Outcome

http://www.vereinoutcome.ch/pdf/outcome_interview_d.pdf 